\title{
Exploiting Body Dynamics for Controlling a Running Quadruped Robot
}

\author{
Fumiya Iida, Gabriel Gómez, and Rolf Pfeifer \\ Artificial Intelligence Laboratory \\ Department of Informatics, University of Zurich \\ Andreasstrasse 15, CH-8050 Zurich, Switzerland \\ Email: [iida, gomez, pfeifer] difi.unizh.ch
}

\begin{abstract}
Exploiting the body dynamics to control the behavior of robots is one of the most challenging issues, because the use of body dynamics has a significant potential in order to enhance both complexity of the robot design and the speed of movement. In this paper, we explore the control strategy of rapid four-legged locomotion by exploiting the intrinsic body dynamics. Based on the fact that a simple model of four-legged robot is known to exhibit interesting locomotion behavior, this paper analyzes the characteristics of the dynamic locomotion for the purpose of the locomotion control. The results from a series of running experiments with a robot show that, by exploiting the unique characteristics induced by the body dynamics, the forward velocity can be controlled by using a very simple method, in which only one control parameter is required. Furthermore it is also shown that a few of such different control parameters exist, each of them can control the forward velocity. Interestingly, with these parameters, the robot exhibits qualitatively different behavior during the locomotion, which could lead to our comprehensive understanding toward the behavioral diversity of adaptive robotic systems.
\end{abstract}

\section{INTRODUCTION}

The use of body dynamics for controlling the robot behavior has recently been explored by many robotics researchers (e.g. [1], [2], [3]) . By exploiting body dynamics, the robots can significantly reduce the computational efforts, which would potentially lead to the significant augmentation of the level of complexity and the speed of the movement. The fundamental problem of this approach, however, is that the desired behavior of the systems is highly dependent on the environmental conditions, which results in the lack of controllability and the diversity of behavior. For example, the Passive Dynamic Walkers can walk down the slope in a very natural way by taking advantage of the body dynamics, but it functions only in a severe constraints of the angle of slope and the ground friction [4], [5], [6]. One of the challenges in this line of research, therefore, is to enhance the controllable behavioral diversity by exploiting the body dynamics.

From this perspective, the rapid legged locomotion is a desirable research topic to explore the use of body dynamics, which requires relatively complex control for the rapid movement. The running mechanisms of legged robots have been successfully engineered during the last couple of decades. The pioneering work by Raibert [7], [8] has shown that the task of a hopping machine can be decomposed into three problems, namely (1) regulating periodic hopping height; (2) maintaining body attitude; and (3) controlling the desired forward speed. Then these control problems can be solved by switching between two control strategies for stance and flight phases. During the stance phase, the robot controls for the problems (1) and (2), and during the flight phase, the problem (3) is dealt with. By following these design principles, it has been shown that monopod, biped, quadruped and hexapod robots were able to maintain the balance and control the forward velocity only by regulating the appropriate angle of attack at touchdown during a flight phase [9], [10], [11], [12], [13]. All these studies are based on a method in which there are two independent control phases, thus the robot needs to identify the flight/stance phase at every computational step by using contact detectors on the feet.

Previously, it has been shown that the rapid quadrupedal locomotion can also be possible with an even simpler mechanism. By exploiting mechanical properties of the elastic legs, a form of quadrupedal running behavior, the so-called bounding gait, was demonstrated without the necessity of global sensory feedback [14]. An interesting characteristics of this control framework is that, even though it utilizes a simple open-loop control, it is possible to achieve the stable periodic gait at the several different forward velocity by changing a single control parameter. Moreover, there are several different such control parameters which vary the quantitative and qualitative locomotion properties such as the hopping height and the gait patterns.

Based on the previous development, this paper presents the control scheme to exploit the body dynamics for the dynamic rapid legged locomotion. By analyzing the relation between the control parameters and the whole body dynamics, we firstly observe a set of behavior variations induced by a simple control method, then it will be used for the purpose of controlling the locomotion behavior. As a result it is shown that one control parameter can regulate the forward velocity, as well as the hopping height and two clearly identifiable different gait patterns. An important conceptual contribution of these experimental results is that the diversity of locomotion behaviors is emerged from the interplay between the body dynamics and the control architecture, which would lead to our comprehensive understanding of the emergence of gaits in biological systems, for example.

The structure of this paper is as follows. In section 2, we 


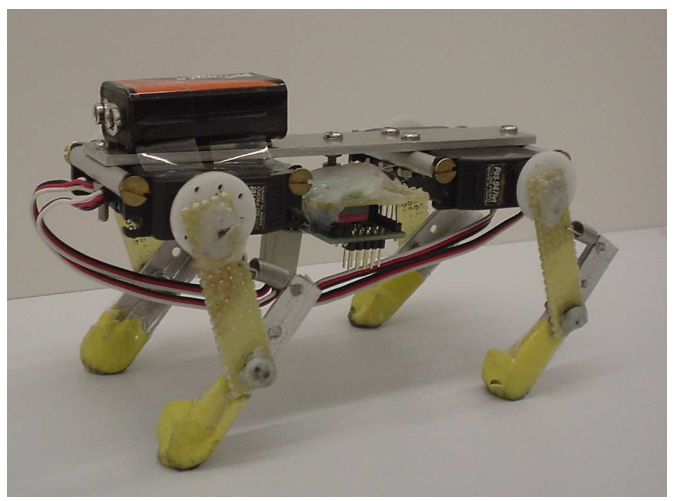

(a)

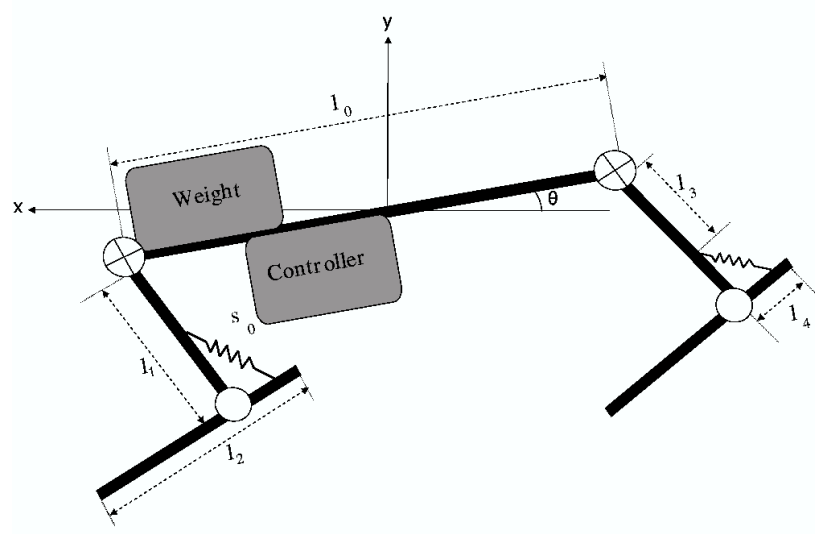

(b)

Fig. 1. (a) A photograph of the quadruped robot. (b) A schematic of the robot. The circles denote passive joints and the circles with a cross inside denote the joints controlled by the servomotors. The specifications of the robot are shown in Table 1.

explain the design and control of our quadruped robot which is used for the experiments described in section 3. Issues leading to further design principles will be discussed in section 4 .

\section{Design And Control of Quadruped Robot}

The use of elastic components in the muscle-tendon system has been investigated in biomechanics (e.g., [15], [16], [17], which leads to the theoretical model of legged animals, the so-called "spring-mass model" [18], [19]. In this model, it was hypothesized that animal's leg could be approximated by a spring loaded inverted pendulum. Interestingly the studies of the spring-mass locomotion models have shown that, due to the self-stabilization mechanisms, rapid legged locomotion can be passive or it requires extremely simple control (e.g. [20] [21]). In this section, we explain the biologically inspired morphological structure and its control strategy which will be used in the following experiments.
TABLE I

THE SPECIFICATION OF THE ROBOT MECHANICAL STRUCTURE

\begin{tabular}{|c||c|c|}
\hline Param. & Description & Value \\
\hline \hline$l_{0}$ & length of body & $142 \mathrm{~mm}$ \\
\hline$l_{1}$ & length of upper leg limb & $42 \mathrm{~mm}$ \\
\hline$l_{2}$ & length of lower leg limb & $56 \mathrm{~mm}$ \\
\hline$l_{3}$ & spring attachment & $15 \mathrm{~mm}$ \\
\hline$l_{4}$ & spring attachment & $20 \mathrm{~mm}$ \\
\hline$s_{0}$ & spring constant & $40 \mathrm{~g} / \mathrm{mm}$ \\
\hline$m$ & mass of the robot & $273 \mathrm{~g}$ \\
\hline
\end{tabular}

\section{A. Morphological Design}

The design of the robot is inspired by the spring-mass model studied in biomechanics. As shown in Figure 1, the robot has four identical legs each of which consists of one standard servomotor (KOPROPO PDS947FET) and a series of two limbs connected through a passive elastic joint. We used aluminum for the design of body frame and legs. The physical dimensions of the robot body are $142 \mathrm{~mm}$ long, $85 \mathrm{~mm}$ wide and approximately $75 \mathrm{~mm}$ high (refer to Table 1 for more detailed specifications). The robot has 4 servomotors located at the shoulders, a micro-controller (Microchip PIC 16F877) and a small weight to adjust the weight distribution of the body, which result in a total weight of $273 \mathrm{~g}$. The control signal for the motors and the electricity are supplied externally through cables. We used the standard serial communication protocol to send the positions of the servomotors from a PC to the micro-controller that produces the modulated signals for the servomotors.

To gain a higher forward velocity, the robot requires higher ground friction. For this reason we have implement a rubber surface at the ground contact in each leg. Although it is difficult to quantitatively measure the slipperiness during dynamic interaction between feet and ground, a good estimate could be the coefficient of friction. The static and dynamic coefficients of friction are approximately 0.73 and 0.55 , respectively.

\section{B. Motor Control}

For the detailed observation of the intrinsic body dynamics derived from the morphological properties, we apply a parsimonious control strategy, in which the controller is kept as simple as possible without sensory feedback at the level of global function. In the following experiments, the motors are controlled by a simple oscillatory position control as follows.

$$
\begin{gathered}
P_{f}(t)=A_{f} \sin (\omega t)+B_{f} \\
P_{h}(t)=A_{h} \sin (\omega t+\phi)+B_{h}
\end{gathered}
$$

where $P_{f}$ and $P_{h}$ indicate the target angular positions of the fore (shoulder) and hind (hip) motors, respectively. $A$ and $B$ determine the amplitudes and the set points of the oscillation, and the frequency $\omega$ and the phase $\phi$ determines the phase delay between these two oscillations of the fore and hind legs. Control of the motors is symmetric in terms of the sagittal plane, i.e. the control of two fore legs is the 


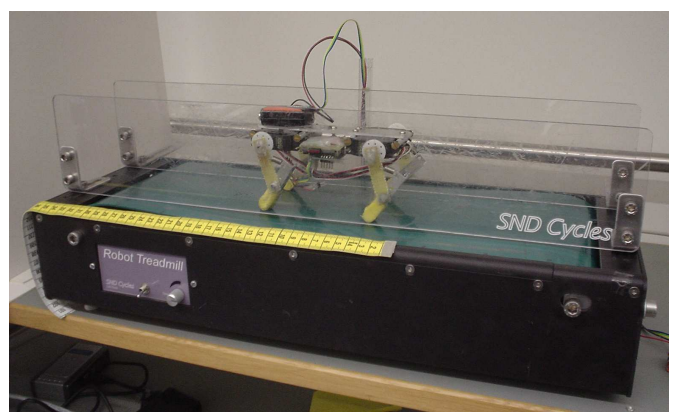

(a)

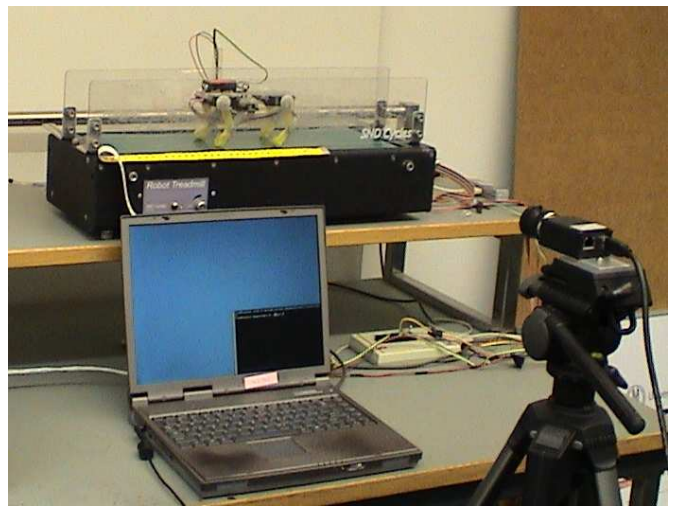

(b)

Fig. 2. (a) The quadruped robot on the treadmill. (b) The experimental setup. Both robot and treadmill are controlled by a laptop computer, and the highspeed camera is installed at the lateral side to observe the running behavior of the robot.

same. The parameters used in the following experiments are heuristically determined as follows. $A_{f}=A_{h}=25$ (degrees), $B_{f}=20$ (degrees), and $B_{h}=10$ (degrees). The control parameters of frequency $\omega$ and phase $\phi$ will be used in the following experiments. The coordinate system of these set points is set to perpendicular with respect to the spine. Note that this control method does not require any global sensory feedback: The controller does not need to distinguish stance and flight phase, the body attitude or leg angles with respect to the absolute ground plane.

\section{EXPERIMENTS}

Despite its simplicity, the control scheme introduced in the previous section exhibits the stable dynamic running behaviors with a set of variations. This section overviews the characteristics of the running behavior and the influence of the control parameters.

\section{A. Experimental Setup}

We used the experimental setup shown in Figure 2, which consists of the robot, a treadmill, a high-speed camera and two sets of computers. The treadmill was developed especially for the robot explained in the previous section, in which the speed of rotational belt can be controlled online by a computer using the USB interface. The size of treadmill is 550(L) x 200(W) x $120(\mathrm{H}) \mathrm{mm}$ (the running surface is $480(\mathrm{~L}) \times 170(\mathrm{~W}) \mathrm{mm}$ ) and
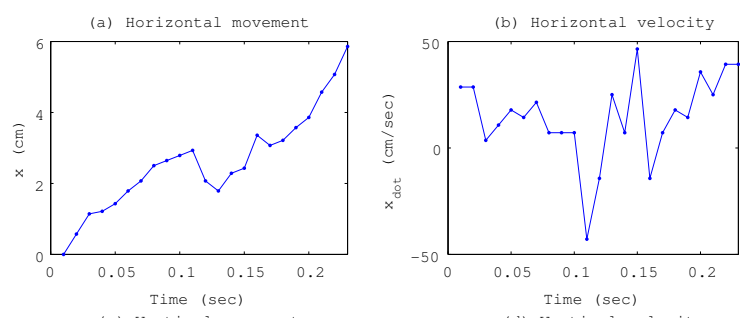

(c) Vertical movement
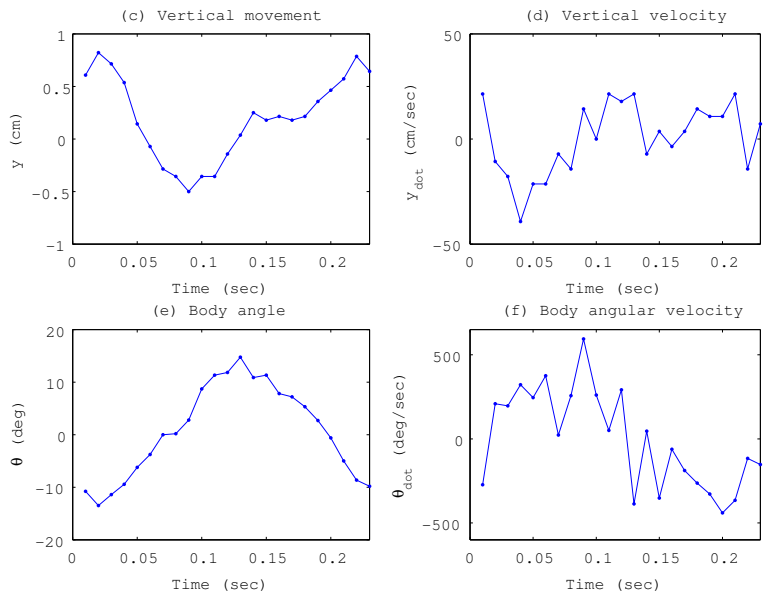

Fig. 3. Time-series changes of the state variables during one leg step, (a) horizontal, (b) vertical, (c) angular movement and their velocity (d, e, f).

the maximum speed of the rotational belt is approximately $60 \mathrm{~cm} / \mathrm{sec}$. This treadmill is controlled by the same computer as the one for the robot such that both are synchronized. Two transparent plastic plates are installed on the top of treadmill to restrict the lateral deviations of the robot during the running experiments.

In this setup, we are able to measure the forward velocity of the robot running in the following manner. With a set of control parameters of the robot, we perform a few running tests and adjust the speed of treadmill such that the robot should run at the center of treadmill. By measuring the speed of treadmill, we estimate the running speed of the robot. In order to analyze the detailed characteristics of the behavior, a highspeed camera is used for the visual analysis from a side view as shown in Figure 2. We used a Basler A602fc (maximum resolution 650x490, frame rate 100fps, IEEE 1394 interface) and the image sequences were stored in a standard PC for the behavior analysis in the later stage. For the behavior analysis, we used a standard visual tracking method, in which an salient visual features on the robot body are extracted in each image, with which we estimate the spatio-temporal behavior patterns of the robot.

\section{B. Intrinsic Stability}

The intrinsic stability of the compliant leg in the feedforward locomotion has been explored previously, although most of them investigated the control scheme with two phases (e.g. [22], [12], [21]). In the first set of experiments, we evaluate 

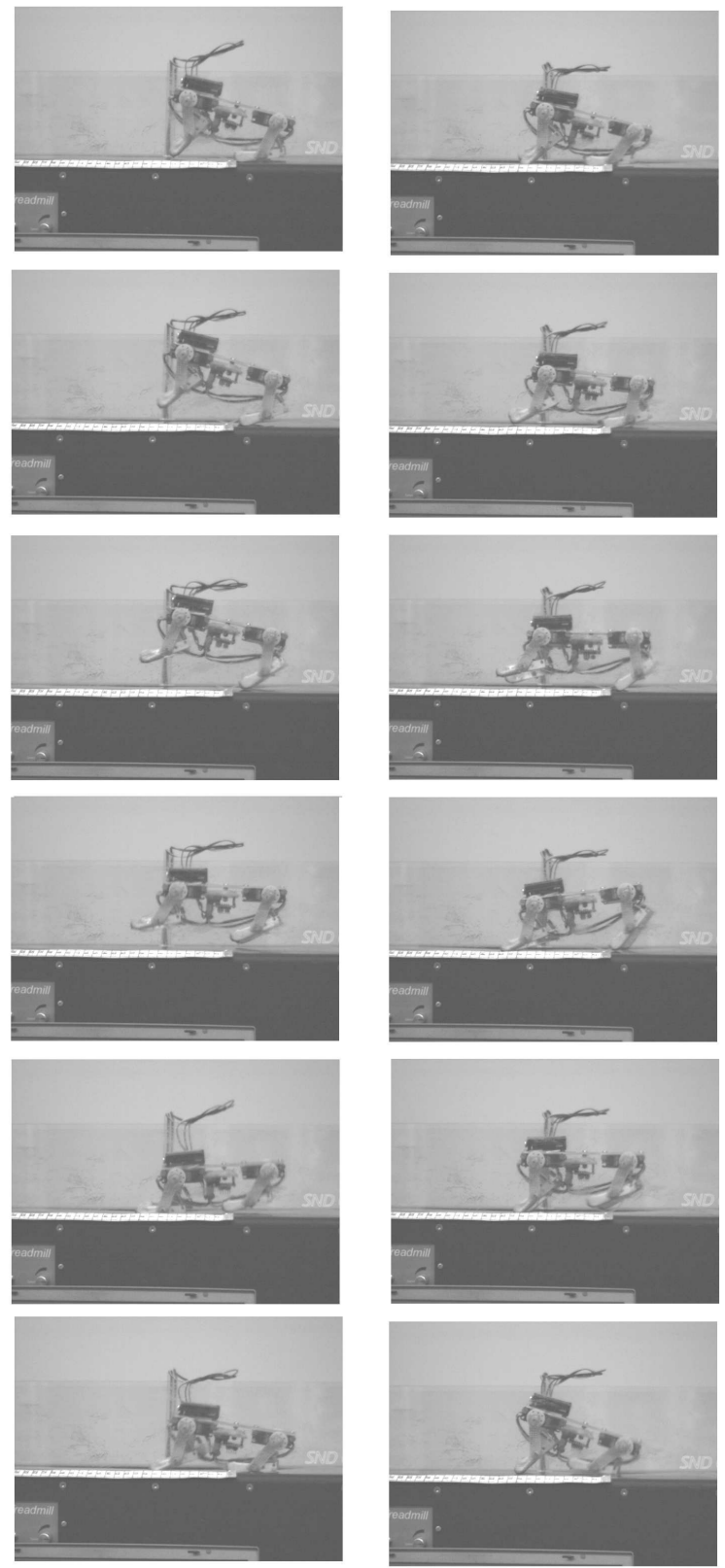

(a)
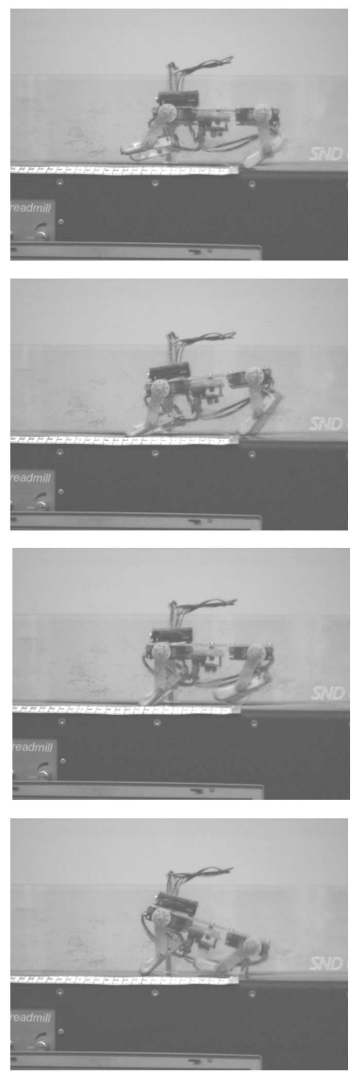

(b)

Fig. 4. A time series photographs during "the gait 0" (a) and "the gait 1" (b). The interval between two pictures is approximately $30 \mathrm{~ms}$.

the stability of the proposed locomotion method without the global sensory feedback. Figure 3 illustrates typical time-series state variables which characterizes the movement of the robot body during one leg cycle. As shown in this figure, all five state variables (i.e. $\dot{x}, y, \dot{y}, \theta$, and $\dot{\theta}$ ) go back to the states at the beginning of the leg step, which can be interpreted as a stable gait cycle.

By searching through the control parameters, we observed two qualitatively different locomotion gaits which are labeled "gait 0" and "gait 1", as shown in Figure 4. In the gait 0 , the hopping height is relatively larger than in the gait
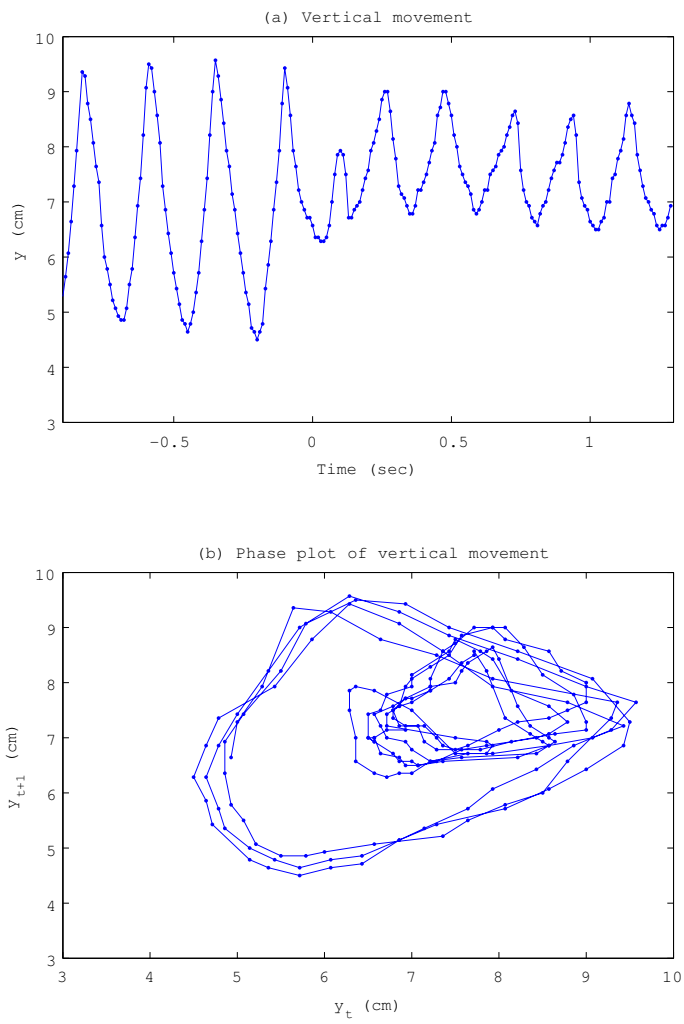

Fig. 5. A typical recovery response from the change of the gait. (a) The timeseries vertical movement of the body is shown before and after the trigger at time $=0$, and (b) its phase plot.

1, which results in the four legs clearly off the ground for some duration in a leg cycle. As explained later in detail, however, the gait 1 generally exhibits larger forward velocity. The intrinsic stability of the proposed locomotion method can be nicely demonstrated when the robot switches between these gaits. A typical response induced by the change of the gait is shown in Figure 5, where the control parameter of phase $\phi$ is varied at time $t=0$. Generally, the periodic gait patterns can be recovered within one or two leg steps. It is important to mention that the change of the control parameters can be at any point in the locomotion cycle, which is a unique property of the proposed control scheme. Note also that a similar recovery response was also observed when an unanticipated irregularity of the ground condition was introduced.

\section{Influence of Control Parameters}

In the next set of experiments, we have analyzed the relation between the forward running velocity and two control parameters, i.e. the frequency $\omega$ and the phase $\phi$ in eq. (1) and (2). As described in the previous subsection, the forward velocity at each control parameter set was estimated by the rotation speed of the treadmill. In this experiment, we have started the running experiment from a standing position of the 


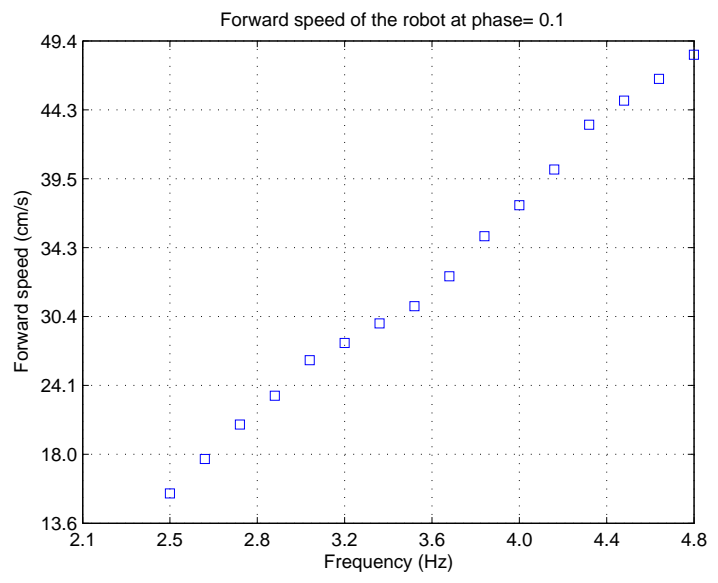

Fig. 6. Average forward velocity of the running robot against the frequency parameter at the phase 0.1 .

robot (i.e. the initial velocity zero), and measured the average forward velocity at each parameter set during 10 seconds. Figure 6 shows the average forward velocity with respect to the frequency parameter at the range of 2.5 to $4.8 \mathrm{~Hz}$, and the phase parameter is always set at 0.1 radians. The stable periodic running behavior is possible over the relatively wide range in terms of the frequency, whereas it was not possible to achieve a stable locomotion when the frequency is lower than $2.5 \mathrm{~Hz}$. The upper range limit is due to the capacity of the motors, therefore it is most likely that the locomotion velocity can go higher. Overall, from this figure, the forward velocity of running behavior varies approximately from 15 to $50 \mathrm{~cm} / \mathrm{sec}$ (from 2 to 7 leg-length/sec) by changing the frequency parameter. It is shown in the figure that the forward velocity increases almost linearly with respect to the frequency parameter.

It is also possible to vary the forward velocity by changing the phase parameter shown in eq. (2). Figure 7 shows the average velocity curves with respect to each phase parameter at three different frequencies. Again we have tested within only the parameter range with which a stable locomotion behavior is possible. Namely it was not possible to maintain a stable gait at the phase parameter, less than -3.0 and over 0.8 radians. These figures show that, by changing the phase parameter, the velocity range covers approximately 15 to 20 $\mathrm{cm} / \mathrm{sec}$. On contrary to the frequency parameter, the curve profiles of forward velocity exhibits the non-linear nature; At the frequency 2.8 and $3.2 \mathrm{~Hz}$, the curve profiles are bell-shape, while the velocity suddenly jumps at the frequency parameter $4.0 \mathrm{~Hz}$.

Interestingly, although both parameters changes the forward velocity, the running behavior is qualitatively very different. As shown in Figure 7, there are two clearly distinguishable preferable states of forward velocity at the frequency parameter $4.0 \mathrm{~Hz}$ in which the transition takes place at the phase of -1.2 radians, where the "gait 0 " has appeared. Particularly, the "gait 0 " generally exhibits the slower velocity and the higher

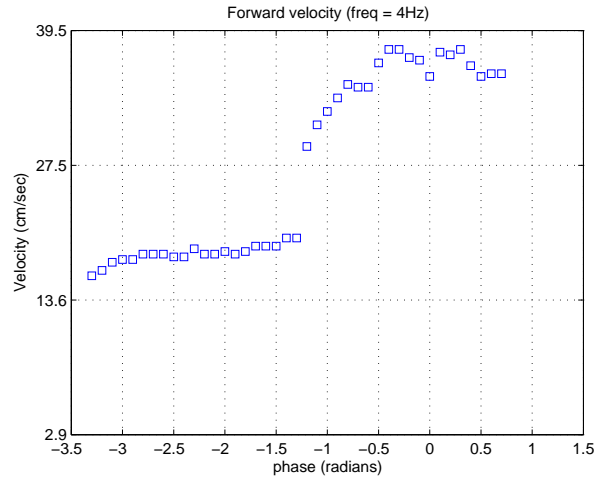

(a)

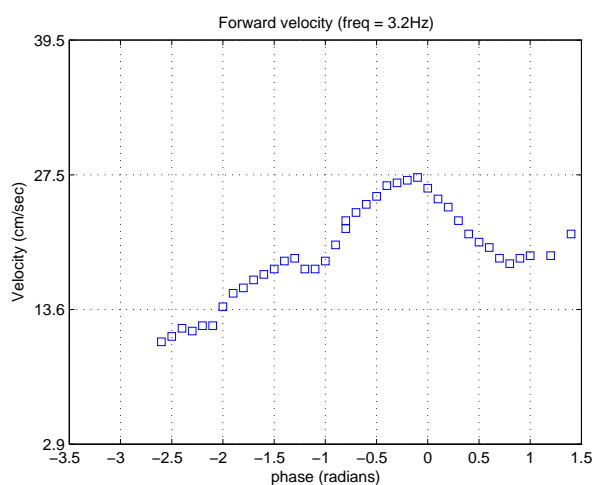

(b)

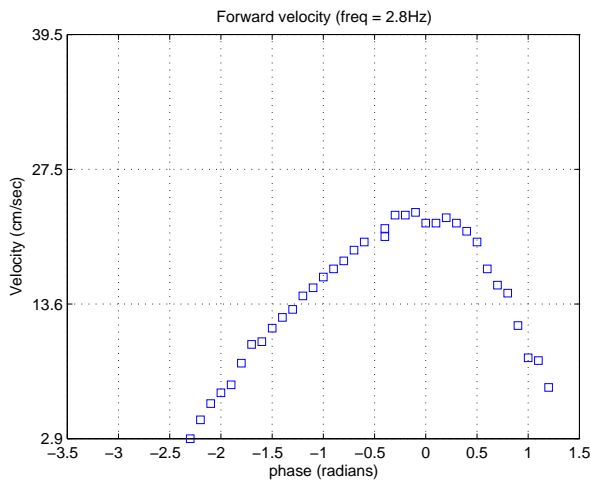

(c)

Fig. 7. Average forward velocity of the running robot against the phase parameter at the frequency parameter (a) 4.0, (b) 3.2 and (c) $2.8 \mathrm{~Hz}$.

hopping, whereas the "gait 1" is faster and lower hoppingheight. Figure 5 more clearly shows the contrast between these two gaits, in which the vertical movement of the robot body during these two gaits obtained from the visual analysis. During the gait 0 , which has been shown to be a slower gait, the robot has higher apex height than the other by approximately $1-1.5 \mathrm{~cm}$. (This is also shown in Figure 4 (a4th frame from top to bottom), where all four legs are clearly off the ground.) In biomechanical terms, the smaller phase value corresponds to the so-called "pronk gait", in which all of the fore and the hind legs swing forward or backward at 

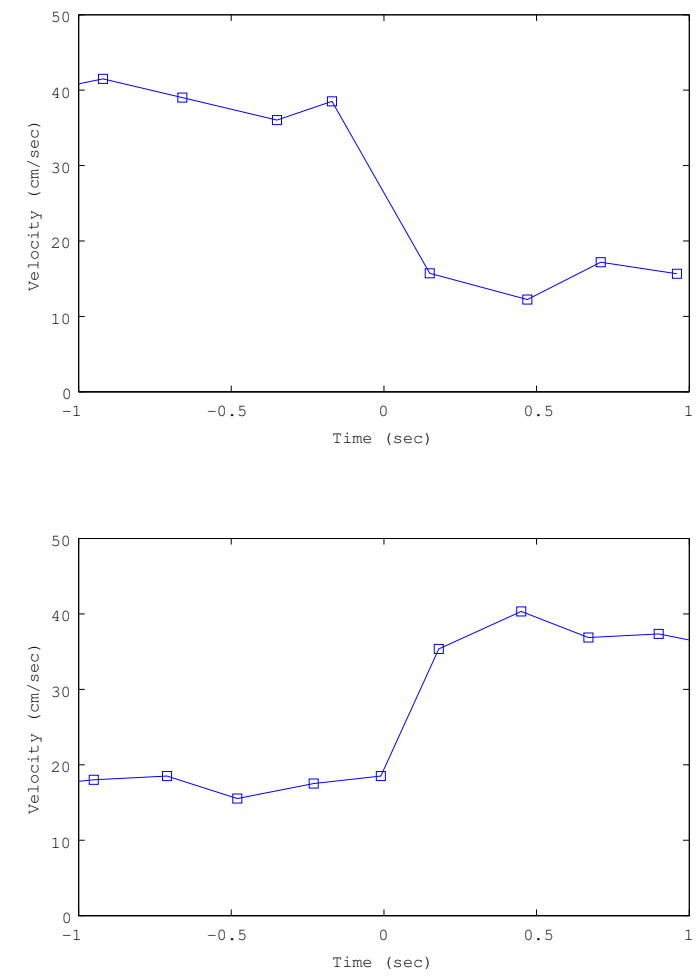

Fig. 8. Transition of the forward velocity when the phase parameter is (a) increased (switching the phase parameter from -1.5 to 0.5 ) and (b) decreased (switching the phase parameter from 0.5 to -1.5 ). The parameter switch is triggered at the time $t=0$.

the same time. On the other hand, the so-called "bounding gait" which corresponds to the large phase value, shows the fore legs and the hind legs swing the opposite direction. Interestingly, animals generally exhibit the pronk gait when they jump higher.

\section{Control of Forward Velocity}

In addition to the steady gait analysis of the locomotion behavior, we performed another set of experiments for switching and regulating between two different forward velocities. In order to test the eminent changes in the velocity, we varied the phase parameter from -1.5 to 0.5 , and keep the treadmill speed at constant through the experiment. Then we conducted the visual analysis, in which we estimated the average forward velocity at each leg step as shown in Figure 8. In both cases of increasing and decreasing the velocity, the forward velocity is generally regulated within one or two steps after switching the parameter as also observed in Figure 5.

\section{DISCUSSION}

On the basis of the experimental results presented in this paper, this section overviews a few conceptual contributions for the control scheme of quadrupedal running behavior.
From the information theoretic viewpoint, this paper explored an additional variation of control method for the quadruped running. Compared to the two-phase control scheme, which is widely employed in many hopping robots (e.g. [7], [8], [9], [10], [12]), the proposed open-loop control scheme introduces three major arguments which could be essential for our comprehensive understanding of legged locomotion. Firstly, the flow of information is unidirectional and there is no signal feedback loop running all through the legs and the body, but the loop is only local, i.e. only in the servomotors. This experimental result achieved by a synthetic investigation could help understanding the physiological nature of legged rapid locomotion. Secondly, the control of speed cannot be computationally simpler than the proposed phase control, because there is no sensory feedback, on the one hand, and the phase is equivalent to a low-pass filter (i.e. a simple time delay), on the other. Thirdly, there are many other parameters such as the frequency, the set points, and the spring constants (if possible) which can potentially control the forward velocity in addition to the phase parameter. The diversity and the flexibility of the proposed control scheme is another interesting aspect to be explored further.

Even though we employed the open-loop control architecture, the underlying mechanism of the locomotion behavior could be the same as the two-phase control scheme; A compliant leg shows the characteristics in which the hopping height and the forward velocity are directly dependent on the leg angle at the touch down as shown in [14]. Based on the characteristics of the compliant legs, the touch-down angle of each leg is explicitly controlled in the two-phase control scheme, but the touch-down angle is self-organized in the proposed control scheme. An interesting outcome of this implicit control of behavior is shown in the experiment of the gait transition (Figure 4, 5 and 7), where the gait transition (from pronk to bound) can be both smooth and intensive; At lower frequency of the leg operation, it seems relatively smooth, but it gives rise to an intensive bifurcation at a higher frequency.

Although we investigated only partially the stability of the periodic locomotion gaits, it is a fundamental issue which needs to be formulated in the future. However, the implications we could obtain from the experimental results are highly interesting when compared to the investigation of legged locomotion in the dynamical systems approach. Initiated by Taga's simulation work [1], it has been shown that the coupling of body and neural system dynamics provides a stability in the legged locomotion [23], [24]. From the experimental results shown in this paper, however, it can be concluded that the purely mechanical dynamics with a simple sinusoidal oscillation is also capable of maintaining the stability.

Although we have explored only a form of rapid locomotion by a minimal control, it does not imply that we don't need sensors, but it demonstrated how much can be achieved with how little control. Particularly, this approach provides additional insight into embodied adaptive behavior or intelligence in general. The control of behavior is quite 
often the major research interest of adaptive locomotion, but the use of body dynamics is also a fundamental mechanism to properly understand behavioral diversity. As illustrated in the case studies of this paper, the functions of the system are no longer separable from the constraints derived from embodiment, if the behavior of the robots highly depends on its body dynamics; there is no longer a clear distinction between hardware and software. In this sense, locomotion behavior is also essential for the high-level cognition, as it enables the agent to construct a "body image" that on the one hand can be used to guide behavior in the real world and on the other as a basis for metaphors on top of which something like cognition can be bootstrapped.

\section{CONCLUSiON}

This paper explored a control method of running behavior of a four-legged robot by exploiting body dynamics. It was shown that a simple design of morphology and control is sufficient for dynamic running behavior with a set of behavioral diversity. The direct contribution of this demonstration is that the use of body dynamics could be potentially very powerful for controlling complex behaviors with rich diversity and the larger speed of the movement. One of the examples is shown as the emergence of the gait transition, in which the smooth and intensive bifurcation between two gaits is observed even in the proposed simple mechanical and control system. Further exploration of this approach would lead to the comprehensive understanding of complex adaptive behavior.

\section{REFERENCES}

[1] Taga, G., Yamaguchi, Y., and Shimizu, H.: Self-organized control of bipedal locomotion by neural oscillators in unpredictable environment. Biological Cybernetics 65 (1991) 147-159.

[2] Yasuo Kuniyoshi, Yoshiyuki Ohmura, Koji Terada, Akihiko Nagakubo, Shin'ichiro Eitoku, Tomoyuki Yamamoto: Embodied basis of invariant features in execution and perception of whole body dynamic actions Knacks and focuses of roll-and-rise motion, Robotics and Autonomous Systems, vol.48, no.4, 189-201, 2004.

[3] Yamamoto, T. and Kuniyoshi, Y. Harnessing the robot's body dynamics: a global dynamics approach, Proc. of 2001 IEEE/RSJ International Conference on Intelligent Robots and Systems (IROS2001), 518-525, 2001.

[4] McGeer, T., Passive Dynamic Walking, The International Journal of Robotics Research, Vol. 9, No. 2, 62-82, 1990.

[5] Collins, S. H., Wisse, M., and Ruina, A.: A three-dimentional passivedynamic walking robot with two legs and knees. International Journal of Robotics Research 20 (2001) 607-615.

[6] Wisse, M. and van Frankenhuyzen, J.: Design and construction of MIKE: A 2D autonomous biped based on passive dynamic walking. Proceedings of International Symposium of Adaptive Motion and Animals and Machines (AMAM03), 2003.

[7] Raibert, M. H., Trotting, Pacing and Bounding by a Quadruped Robot $J$. Biomechanics, Vol. 23, Suppl. 1, 79-98, 1990.

[8] Raibert, H. M., Legged Robots That Balance, The MIT Press, 1986.

[9] Poulakakis, I., Smith, J. A. and M. Buehler On the Dynamics of Bounding and Extensions Towards the Half-Bound and the Gallop Gaits, In: Proc. of the 2nd Int. Symp. on Adaptive Motion of Animals and Machines, 2003.

[10] Buehler, M. Battaglia, R., Cocosco, A., Hawker, G., Sarkis J., and Yamazaki K., Scout: A simple quadruped that walks, climbs and runs In: Proc. Int. Conf on Robotics and Automation, 1707-1712, 1998.

[11] Murphy, K. N. and Raibert, M. H. Trotting and bounding in a planar two-legged model, Theory and Practice of Robots and Manipulators, Morecki, A. et al. (Eds.), 411-420, 1984.
[12] Hyon, S., Kmijo, S., Mita, T., 'Kenken' - A Biologically Inspired OneLegged Running Robot J. of the Robotics Society of Japan, Vol. 20, No. 4, 453-462, 2002.

[13] Cham, J. G., Bailey, S. A., Clark, J. E., Full, R. J. and Cutkosky, M. R. Fast and robust: hexapedal robots via shape deposition manufacturing, The International Journal of Robotics Research, 21, Issue 10, 869-882, 2002.

[14] Iida, F. and Pfeifer, R., "Cheap" rapid locomotion of a quadruped robot: Self-stabilization of bounding gait, Intelligent Autonomous Systems 8, F. Groen et al. (Eds.), IOS Press, 35, 642-649, 2004.

[15] Cavagna, G. A., Heglind, N. C. and Taylor, C. R. Mechanical work in terrestrial locomotion: Two basic mechanisms for minimizing energy expenditure, American Journal of Physiology 233, R243-R261, 1977.

[16] Alexander, R. McN., Elastic Energy Stores in Running Vertebrates, Amer. Zool., 24, 85-94, 1984.

[17] Alexander, R. McN., Three uses for springs in legged locomotion, The International Journal of Robotic Research, 9, No. 2, 53-61, 1990.

[18] McMahon, T. A., Cheng, G. C., The Mechanics of Running: How Does Stiffness Couple with Speed?, J. Biomechanics, Vol. 23, Suppl. 1, 65-78, 1990.

[19] Blickhan, R. The spring-mass model for running and hopping, $J$ Biomechanics 22:1217-1227, 1989.

[20] Kubow, T. M., Full, R. J., The role of the mechanical system in control: a hypothesis of self-stabilization in hexapedal runners, Phil. Trans. $R$. Soc. Lond. B, 354, 849-861, 1999.

[21] Seyfarth, A., Geyer, H., Guenther, M., Blickhan, R., A movement criterion for running, Journal of Biomechanics, 35, 649-655, 2002.

[22] Poulakakis, I., Papadopoulos, E., and Buehler, M. On the Stable Passive Dynamics of Quadrupedal Running, 2003 IEEE Int. Conf. on Robotics and Automation (ICRA), 2003.

[23] Kimura, H., Fukuoka, Y., Hada, Y., Takase, K., Three-dimensional Adaptive Dynamic Walking of a Quadruped - rolling motion feedback to CPGs controlling pitching motion -, Proc. of the 2002 IEEE International Conference on Robotics and Automation, 2228-2233, 2002.

[24] Fukuoka, Y., Kimura, H., and Cohen, A. H., Adaptive dynamic walking of a quadruped robot on irregular terrain based on biological concepts, The International Journal of Robotics Research, Vol. 22, Issue 3, 187-202, 2003. 